\title{
ESTRATEGIAS INNOVADORAS PARA EL DESARROLLO ECONÓMICO EN TIEMPOS DE CRISIS
}

\section{INNOVATIVE STRATEGIES FOR ECONOMIC DEVELOPMENT IN TIMES OF CRISIS}

\section{ESTRATÉGIAS INOVADORAS PARA O DESENVOLVIMENTO ECONÓMICO EM TEMPOS DE CRISE}

Luis Aldimir Canchingre Bone ORCID: https://orcid.org/0000-0001-7295-6552 Universidad Técnica "Luis Vargas Torres" de Esmeraldas luis.canchingre@utelvt.edu.ec Esmeraldas- Ecuador

Romina Tanaga Oyarvide Ibarra ORCID: https://orcid.org/0000-0001-6073-7360 Universidad Técnica "Luis Vargas Torres" de Esmeraldas romina.oyarvide@utelvt.edu.ec Esmeraldas- Ecuador

Ivan Octavio Endara Argüello ORCID: https://orcid.org/0000-0003-2088-9161 Universidad Técnica "Luis Vargas Torres" de Esmeraldas ivan.endara@utelvt.edu.ec

Esmeraldas- Ecuador

Edwin Daniel Moran Villamarin ORCID: https://orcid.org/0000-0003-2136-9600 Universidad Técnica "Luis Vargas Torres" de Esmeraldas edwin.moran.villamarin@utelvt.edu.ec

Esmeraldas- Ecuador

Diagramación e ilustración portada Sindy Catherine Charcas Ibarra 


\section{RESUMEN}

El presente artículo tiene como propósito determinar estrategias de innovación para el desarrollo económico en tiempo de crisis, para lo cual se ha realizado una revisión de literatura relacionada en el tema, haciendo énfasis en las evidencias encontradas en América Latina y específicamente, en Ecuador. El tipo de investigación es cualitativa con apoyo de la revisión bibliográfica documental. Se concluye que la innovación es un proceso complejo vinculado al emprendimiento, a la capacidad creativa y da paso a una estrategia de desarrollo económico. Se considera la importancia del uso de tecnologías de información y comunicación, como tendencia gerencial de transmisión de conocimiento que promueve innovación y acelera cambios en el sector económico.

Palabras clave: innovación, desarrollo económico, tiempo de crisis.

\section{ABSTRACT}

The purpose of this article is to determine strategies for innovation and economic development in times of crisis, for which a review of related literature on the subject has been carried out, emphasizing the evidence found in Latin America and specifically, Ecuador. The type of research is qualitative with the support of the documentary bibliographic review. It is concluded that innovation is a complex process linked to entrepreneurship, creative capacity and gives way to an economic development strategy. Regarding economic development strategies, the importance of the use of information and communication technologies is considered as a managerial trend for the transmission of knowledge that promotes innovation and accelerates changes in the economic sector.

Keywords: innovation, economic development, time of crisis.

\section{SUMARIO}

O presente artigo destina-se a identificar estratégias de inovação para o desenvolvimento económico em tempo de crise, tendo sido efectuada uma revisão da literatura relacionada com o tema, enfatizando as evidências encontradas na América Latina e especificamente no Equador. O tipo de pesquisa é qualitativa com apoio da revisão bibliográfica documental. Conclui-se que a inovação é um processo complexo ligado ao empreendedorismo, à capacidade criativa e dá lugar a uma estratégia de desenvolvimento económico. Considera-se a importância do uso de tecnologias de informação e comunicação, como tendência gerencial de transmissão de conhecimento que promove inovação e acelera mudanças no setor econômico.

Palavras chave: inovação, desenvolvimento económico, tempo de crise. 


\section{Introducción}

En tiempos de crisis, el impacto económico es más profundo y exigente que en otros escenarios a la hora de aplicar correctivos a nivel de esfuerzo y gasto. Plantea Álvarez (2013), la innovación juega un papel protagónico en el sentido de focalizar las posibilidades de avance que se asoman en un mundo disgregado, dinámico y cambiante. Se hace necesario entonces, una investigación que estudie esa realidad emergente y que descubra alternativas viables y posibles, cuyo énfasis sea construir economías que den respuesta a los nuevos retos de un proceso diversificado, como el actual. El presente estudio orientado a estrategias innovadoras para el desarrollo económico en tiempo de crisis, es resultado de investigaciones ejecutadas en la Facultad de Ciencias Administrativas y Económicas de la Universidad Técnica "Luis Vargas Torres" de Esmeraldas, los cuales arrojaron ideas de desarrollo económico en momentos donde las condiciones salen de su foco y se establecen nuevos mecanismos de acción para el dinamizar los procesos empresariales.

Un aspecto importante a considerar en este artículo es el estudio de algunos enfoques teóricos, herramientas o estrategias que despejen el camino hacia la modernización de los estándares de calidad basados en la innovación. Pues como lo advierte Blanco et al. (2016), esta modernización de conceptos y teorías para aplicar el nuevo modelo de desarrollo, se ancla en una trayectoria variada y compleja de aciertos y fracasos resultantes de un modelo agotado, que urge de cambios y que por ser de carácter emergente conlleva una serie de tácticas, estrategias de choque que posibiliten sobre la marcha, otras transformaciones basadas en la innovación.

Así, las crisis profundas con sus consecuentes degeneraciones a nivel económico, se centran en reinventar el empleo o las actividades lucrativas que sostienen el aparato productivo de una sociedad. Señalan Pinedo y Pitre (2017), la pérdida de perspectivas, de empuje y motivación incita a la búsqueda de nuevos paradigmas, resultando fructífera la tarea de moldear la reconstrucción del modelo productivo partiendo de una fase de transición entre un enfoque insostenible, inoperante y agotado y la nueva propuesta, alternativa exploratoria y novedosa que busca el renacimiento de una economía urbana. Economía que debe responder a las exigencias del nuevo orden en lo económico, con todas las relaciones concomitantes que le rodean.

De este modo, la sociedad actual debe sentar las bases de adaptación hacia la complejidad de un contexto basado en la competitividad de enfoques anglosajones de atención y modelación educativa, que paulatinamente sorprenden al escenario de la crisis. Por lo tanto, diversas investigaciones han antecedido el componente epistémico acá planteado, entre ellos, Méndez (2016), denotando la importancia de asumir una posición basada en la innovación, para enfrentar la demanda de la crisis política, económica y social que caracteriza los tiempos actuales.

En este sentido, juega un papel relevante la innovación, como emprendimiento dinámico que surge para fortalecer propuestas de valores, cuidar la liquidez en un determinado contexto y momento para revitalizar las economías urbanas y lograr que las nuevas sociedades sean inclusivas y sostenibles en el tiempo (Subirats y García, 2015). En los actuales momentos, a nivel mundial está planteada una infinidad de propuestas para reinventarse a partir de cambios profundos, lo cual puede convertirse en una fortaleza, la aceleración digital y su sostenibilidad en el tiempo, es un gran reto.

De igual manera, es necesaria la generación de soluciones y la atención de desafíos económicos de sostenibilidad, dando respuestas a corto plazo a las situaciones del entorno, como el caso de Ecuador por ejemplo, que en 40 días con un equipo multidisciplinario logró atender la contingencia presentada a partir de la pandemia Covid-19, reacción inmediata e innovadora, que permitió validar y adaptar equipos para ponerlos al servicio del público porque cuando no existe el insumo adecuadooestánestablecidoslosnexos paraconseguirlos, de igual manera hay que responder. El ecuatoriano, es un caso donde la empresa salió fortalecida y no liquidada ante la crisis, como lo indican Camargo et al. (2017). Por lo tanto, Innovación es cambiar el rendimiento de los recursos, transformando el valor y modificándolo satisfactoriamente para el consumidor.

Otro elemento importante que se debe sumar al debate, es la importancia que refleja la visión de empuje y motivación del talento humano como eje transversal de la innovación, advertida como una nueva forma de hacer las cosas a través del uso de la capacidad instalada, medio y recursos, pero con un método distinto, que por tal modificación es interesante. Ello redunda en nuevas formas de pensamiento, formas de trabajo y verificación de resultados, sin dejar de mencionar la importancia del medio donde opera la transformación, que normalmente presenta la oportunidad de equilibrio en el sistema.

En este orden de ideas, surge el emprendimiento como expresión de la innovación, mediante el cual se aprovechan las virtudes y cualidades humanas para la generación de recursos y la promoción del empleo, con aporte significativo a la capacidad de compra y poder adquisitivo, promoviendo una ventaja competitiva a los índices microeconómicos y un apuntalamiento hacia el estado de satisfacción que continuamente vincula capacidad humana, virtudes y destrezas. 
De este modo, el presente artículo tiene como propósito estudiar estrategias innovadoras y desarrollo económico en tiempo de crisis.

\section{Marco teórico}

\section{Innovación y Desarrollo}

El potencial innovador se puedever limitado en el camino hacia el desarrollo debido a muchos factores negativos que caracterizan los espacios dominados por la globalización y la falta de regulación de los mercados, así como la resistencia al cambio, en la gran mayoría de consumidores. Es por ello que las claves para el éxito, según Ycaza y Sánchez (2016), sería repotenciar los conocimientos, estimular la creatividad, promover cambios de base, explorar otras estructuras más equilibradas para los fines establecidos. Según Caridad et al. (2018), aquí es donde entra la resiliencia como estrategia perfecta para superar y adaptarse a los cambios.

Siendo la innovación un proceso emergente para enfrentar las crisis, debe apoyarse necesariamente en una tradición, un motor importante para el desarrollo, producto de la dinámica mundial y el cambiante universo del mercado, por ello, aportan Hernández y Platero (2012), que se ha transformado dando paso a la necesidad de crear una nueva competitividad empresarial y nuevos retos. Esta visión tiene un alto contenido subjetivo porque parte de la capacidad de adaptarse a situaciones difíciles, aprendiendo a salir a flote, impulsando nuevos procesos de aprendizaje individual y colectivo en una suerte de inteligencia compartida, procurando una mirada transversal en el sentido de ver qué están haciendo otros, librando obstáculos que son culturales básicamente y teniendo muy presente que aquellos que no pudieron superar las dificultades tendrán que resignarse a ser perdedores (Mazin, 2021).

La innovación puede surgir de cualquier parte y originarse de una situación o producto aparentemente irrelevante, pero con la chispa de la creatividad activada puede convertirse en un elemento de arranque, de sustento a grandes soluciones y proyecciones atractivas para el crecimiento y desarrollo económico. Los elementos básicos para que una estrategia funcione, según Camargo et al. (2017), son:

- El reto interno, creer en uno - tomar acción.

- Encontrar la diferencia, la condición que va a hacer único el producto, el bombardeo de estímulos que haga a diario.

- Identificar la diferencia que tiene nuestro producto con respecto a los demás.

- Conectarse emocionalmente con el consumidor a través del mensaje.

- Posicionarse como experto o autoridad a través de la tecnología, material gratuito de difusión reciprocidad agrega valor.

- Hacer alianzas estratégicas con la competencia es muy diversa.

- Buscar aliados y establecer convenios de ayuda mutua para posicionarse en el mercado estableciendo relaciones comerciales de manera amigable, integradora mirando en el otro competidor a un cómplice y no un rival. En este sentido el resultado será el fortalecimiento de la relación comercial con la búsqueda constante de mecanismos modernos.

- Idear una estrategia de venta no significa manipular o engañar deliberadamente la conducta o conciencia del comprador.

De este modo, si un artista puede convertir una piedra en una escultura de belleza insospechada, de la misma manera un emprendedor ávido de éxito puede crear fórmulas y estrategias de ventas y aplicarlas oportuna y adecuadamente para transformar el mundo a su favor para generar nuevas propuestas de negocio (Guevara, 2020). Si toda conducta humana obedece a programaciones conscientes o inconscientes en el cerebro, luego que los cinco sentidos han sido impactados o impresionados por el mundo que los rodea, viene obligatoriamente una respuesta a ese estímulo. De allíque el vendedor de una idea o de un producto se apoye en la estrategia de persuasión.

Partiendo del hecho de que todo cliente es el objetivo primordial del proceso, es decir, el principio y fin de todo negocio, pues es en torno a él deben girar todas las estrategias planificadas, para que un cliente se convierta en consumidor debe ser complacido en tres aspectos que según Munduate et al. (2014) son:

- Cumplir un objetivo

- Satisfacer una necesidad

- Cumplir un deseo

\section{Figura 1}

Criterios referentes a las variables de innovación (Zurbriggen y Lago, 2020).

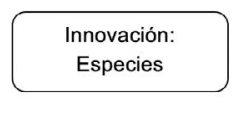

\begin{tabular}{|c|c|}
\hline Social & $\begin{array}{l}\text { - Beneficia a un grupo significativo de } \\
\text { personas }\end{array}$ \\
\hline Vigente & Es reciente y actual \\
\hline Transformadora & $\begin{array}{l}\text { Tiene impacto en relación con variables de } \\
\text { desarrollo social (costos, calidad de vida, gestión, } \\
\text { ingreso, empleo) } \\
\text { - Ha superado la fase de } \\
\text { experimentación }\end{array}$ \\
\hline Consolidada \\
\hline Genuina & $\begin{array}{l}\text { Emerge de los propios interesados en } \\
\text { un proceso verificable }\end{array}$ \\
\hline Original & $\begin{array}{l}\text { No se trata de que sea una experiencia repetida } \\
\text { pero no es necesario que sea un invento }\end{array}$ \\
\hline Expansiva & $\begin{array}{l}\text { Podría ser reproducida en otro lugar a } \\
\text { mayor escala }\end{array}$ \\
\hline
\end{tabular}


Tal como lo señala Zurbriggen y Lago (2020), el impacto social se aprecia en la novedad de los procesos, métodos y estrategias para generar recursos, inversión y solidez. Motivar la creatividad en el terreno económico teniendo como base la estructura de crisis, no es tarea fácil, sin embargo, opina Sánchez (2020), el factor humano siempre encuentra las maneras de ubicar fortalezas en situaciones adversas. La innovación tiene el oficio específico de emprender; en una institución de servicio público, en una compañía existente o en una nueva empresa, es el recurso que el empresario utiliza para crear nuevos bienes y generar abundancia.

ComoloseñalaPimiy Rafini(2015), existeninnovaciones que nacen de cualidades ingeniosas, aunque la mayoría de las innovaciones exitosas resultan de una indagación consciente y deliberada de oportunidades difíciles de encontrar. El ser humano está permanentemente insatisfecho y anda en una búsqueda interminable, es allí donde surge entonces la tarea titánica de mantener vivo el espíritu innovador en el sistema de producción.

Según Zurbriggen y Lago (2020), existen cuatro áreas de oportunidades dentro de una organización:

\section{Figura 2}

Oportunidades organizacionales (Zurbriggen y Lago, 2020).

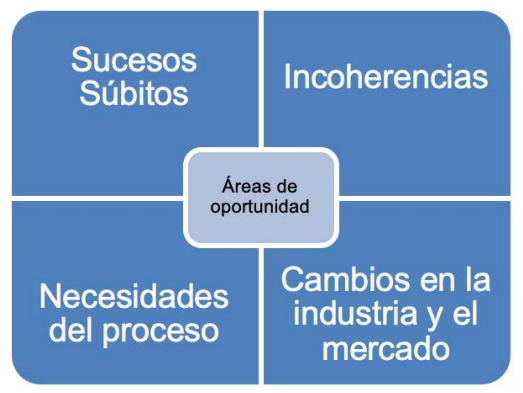

También señala Zurbriggen y Lago (2020), existen fuentes adicionales de oportunidad fuera en un medio social:

\section{Figura 3}

Fuentes adicionales de Oportunidad (Zurbriggen y Lago, 2020).

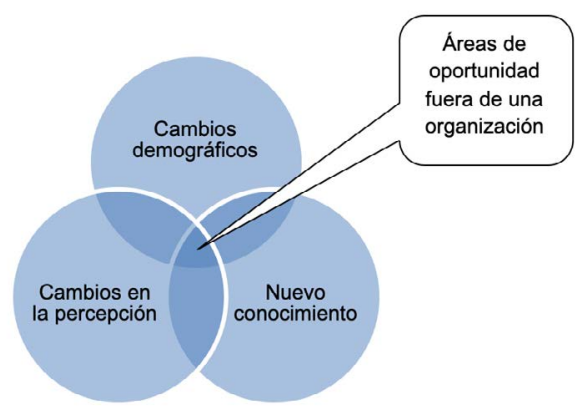

\section{Innovación: Estrategias de Vanguardia}

Incorporar la herramienta de la innovación como variable independiente para mejorar procesos y resolver problemas implica también sortear las limitaciones preexistentes en el camino hacia el desarrollo, debido a factores negativos que dominan los espacios guiados por la globalización y la falta de regulación de los mercados, así como la innegable resistencia al cambio. Las brechas que existen entre los países desarrollados y los países en vías de desarrollo son muy importantes a nivel de presupuesto. En este sentido, indica Umaña et al (2018), a nivel de presupuesto, Alemania, Japón, Estados Unidos, China, Israel, entre otros manejan unos sobre el 2, 3 y 4\%, en cambio en América Latina la asignación no rebasa el $1 \%$.

En el año 2018, Ecuador asignó un escaso 0,43\% de presupuesto al emprendimiento, obviamente con ese respaldo económico la investigación no se activa enérgicamente, por lo que se hace necesaria la incorporación del sector privado y las áreas gubernamentales para que la investigación tenga soporte garantizado y monitoreo continuo. A la par de ello, hay que lidiar con empresas que no quieren competir porque como se resisten al cambio, prefieren acomodarse y reincidir en prácticas tradicionales de mercadeo a una esperanza de éxito a largo plazo. El desarrollo y la innovación tienen un costo y además amerita de tiempo suficiente para alcanzar la experiencia (Goldenberg, 2018).

Innovar implica agregar, incorporar algo nuevo, un elemento que no existió y que se supone que va a mejorar lo existente. Es el proceso donde el ser humano transforma lo que tiene en sus manos para generar una herramienta de trabajo productiva. En Ecuador, según Umaña et al (2018), existe un alto índice de empresarios que no ha internalizado aun el concepto de innovación y por ello se niega o se resiste a apoyarse en ella. No obstante, el ecuatoriano actualmente está incursionando en una nueva capacitación y búsqueda de otros escenarios y experiencias que repotencien su mercado.

Según González y Romero (2018), Ecuador tiene una tasa importante de emprendimientos, pero por estar destinadosacomercios minoristas, hoteles yrestaurantes, rubros que no representan solidez económica ni agrega valor al insumo, carece de importancia y no resuelve el desafío que exige el mundo competitivo de la economía. Es necesario entonces entender que Ecuador tiene la infraestructura para el desarrollo, tiene los empresarios dispuestos a hacer el trabajo, pero carecen de la preparación en términos de competir con valor agregado, innovando rompiendo paradigmas.

El concepto de innovación en términos económicos y de sostenibilidad en el ámbito moderno ha cobrado una vigencia importante. Las oportunidades de éxito en este contexto están determinadas por los recursos 
y la potencialidad de las regiones, así como también por las brechas culturales y sociales que condicionan obligatoriamente la toma de decisiones. La época dorada, donde las grandes compañías multinacionales eran las que marcaban la pauta en las actividades lucrativas de las comunidades, ya pertenece a la historia, dando paso a una economía de carácter autónomo donde aparece el emprendimiento con sus consiguientes características. Este surgimiento del emprendedor como nueva iniciativa económica se ha convertido en el fenómeno mundial, enfatizando en Europa y Latinoamérica su anclaje (Rodríguez, 2021).

González (2021), la innovación como el motor del cambio económico y también la concibe como una destrucción creativa que lleva en su interior una diferenciación entre "invención" e "innovación". Para él, el titulo de emprendedor sólo lo ostentan aquellos que son capaces de innovar constantemente, de ver más allá de su entorno sociocultural. Para Villegas (2021), la innovación es una nueva manera de hacer las cosas que se someten a la consideración de un público consumidor, por lo tanto, se convierte en una estrategia de competitividad que tiene el éxito asegurado si se hacen bien las cosas (Elisondo y Donolo, 2013). A nivel social también la innovación tiene un rol protagónico sobre todo para enfrentar situaciones complejas que se puedan presentar en el ámbito de la salud, educación, medioambiente, entre otros, donde las estrategias convencionales ya no tienen cabida porque los escenarios cambiaron.

Al respecto, conciben Martínez et al. (2021), la innovación social como la estrategia que permite desarrollar nuevas formas de administrar y echar a andar empresas con la ayuda activa de los interesados en el cambio. Para Zurbriggen y Lago (2020), la innovación significa una solución emergente a un problema que puede ser de producto o de proceso. La solución de esos problemas que se presentan en la colectividad permite que las sociedades sean más equilibradas y más equitativas.

Para países como Chile, Colombia, Ecuador, Venezuela, en general, toda Latinoamérica, el entorno social ha pasado a ser un problema de primer orden, debido a la desigualdad, falta de inclusión, empleo informal, poca credibilidad en los entes de gobierno, poca o nula sustentación legal para los negocios, es imperativo trabajar en la búsqueda de soluciones efectivas y de gran impacto a corto y mediano plazo y que propongan las bases para que sigan surgiendo personas que enfrenten con valor real todas las contingencias que se puedan presentar.

Para Magomedova et al. (2020), la innovación es la creación, aceptación e implementación de nuevas ideas que nacen, se desarrollan y se van concretando hasta su total aceptación, logrando transformar un proceso o salir de un quiebre. Cuando la estrategia innovadora no funciona o fracasa, se debe a muchas razones entre las cuales podemos mencionar: repetición de errores, falta de inversión, entre otros. Actualmente toda empresa apuesta por innovar pero muchas veces incurren en intentos fallidos porque se resisten a realizar cambios relevantes en su patrimonio.

Toda innovación, sea de la motivación que fuere tiene un ciclo de vida que, según Magomedova et al. (2020), coincide con el de las organizaciones: puesta en marcha, crecimiento, madurez y declive, lo que hay que cuidar es que se mantenga en el tiempo, que se convierta en un proceso constante y que evolucione en la medida que avance la organización y que se mantenga en vigencia. Cuando la innovación social aglutina diversos sectores de la sociedad, los interrelaciona para beneficio del colectivo, entonces origina la mejor y más exitosa estrategia de desarrollo económico.

Según Magomedova et al. (2020), la innovación tiene dos dimensiones: la novedad y el mejoramiento. La novedad se refiere a las propuestas inferidas de la necesidad de dar solución a un problema o conflicto, debe ser contundente y eficaz y promover el mejoramiento que vendría a ser la solución, el desenlace o la idea llamativa para lograr cambios de conducta por la satisfacción de una necesidad. El término innovación es asumido por diferentes autores como una solución, una estrategia, un proceso, un cambio, una práctica, pero también está ligada al término emprendimiento, cuya acepción más oportuna es la capacidad de asumir riesgos, de inventar, plantear nuevas ideas para crecer y renovar, porque para el emprendedor los problemassonoportunidades deavance.

En las últimas décadas la sociedad civil ha demostrado más protagonismo en la búsqueda de soluciones a problemas locales. América Latina a pesar de poseer muchas fortalezas culturales y naturales, cuenta con situaciones de quiebre, consistentes en exigencias y reclamos de la población porque el mercado y el sector público no ha cubierto expectativas generadas por pobreza extrema, poca inclusión social, problemas muy marcados y de larga data que la sociedad reclama muy justamente sean resueltos ya. A pesar de grandes esfuerzos y los consiguientes logros de unos entes interesados en materia económica, falta mucho por hacer en el tema de la insatisfacción de las necesidades básicas, lo que se ha logrado, en este sentido, es muy precario o de baja calidad (Magomedova et al., 2020).

Han surgido en América Latina las Ilamadas Antenas de Innovación Social que son organizaciones dedicadas al asesoramiento técnico para promover actividades de innovación apoyando las redes sociales y los diferentes emprendedores (Buckland et al. ,2014, como se citó en Medina, 2020). 
Según González y Romero (2018), Ecuador es uno de los países latinoamericanos que anda en busca de mejorar su calidad de vida a través del emprendimiento innovador, así nace la $\mathrm{AEI}$ que es una Alianza para el Emprendimiento y la Innovación, con incentivos monetarios y tributarios para susempresarios.Destacael informequelamejor propuesta en el área de innovación la tiene el entrenamiento formal para el recurso humano, sin embargo, los resultados de este proyecto aún no están analizados por lo que se sigue afirmando que en Ecuador existe muy poca innovación. Una de las razones es la poca integración entre el sector privado y los entes gubernamentales.

Es el Estado el que debe propiciar una alianza para el desarrollo nacional, a través de emprendimientos locales con miras al alcance de una economía afianzada en su diversificación, cuyo financiamiento debe ser compartido entre el ente privado y el estado. Crear el cambio social que amerita la sociedad actual, articulando los diferentes sectores de la sociedad civil, en procura de un desarrollo. Para ser competitivo en un mercado internacional tan exigente, disperso y cambiante, las sociedades deben contar y apoyarse en la tecnología para garantizar el éxito y el progreso, porque de nada sirve un emprendimiento bien planificado sin la ayuda de mecanismos de tecnología adecuados para su difusión y ejecución (González y Romero, 2018).

Actualmente, debido a la globalización, a nivel económico se puede hablar de una verdadera revolución de mercadotecnia. Esta nueva visión de negocios concibe y plantea la reagrupación de actividades que comprometan y graben en la mente del consumidor los posibles deseos y necesidades que lo inciten al consumo. Tal como lo señala Zurbriggen y Lago (2020), desde el punto de vista sociológico la mercadotecnia tiene una inclinación más humanística, entendiendo que no se puede vender todo lo que se le ocurra a un determinado comercio sino producir lo que el consumidor quiere comprar o necesita, o solicita América Latina actualmente se encuentra sumergida en una crisis global motivado a diferentes causas, que van desde cambios políticos, caída del petróleo, inclusión social, hasta la pobreza extrema con todo lo negativo que ella acarrea. Este panorama tan poco halagador obliga a repensar el futuro.

Las ventas, por ejemplo, son estrategias de consumo que sustentan su existencia en el intercambio mutuo entre el bien y su equivalente en términos monetarios. El valor de esta técnica radica en la satisfacción de necesidades y deseos de una población que exige. Si el creativo cree algo, su inconsciente se abocará a hacerlo realidad a pesar de las adversidades y las condiciones externas, discordes con el deseo, esa creencia se concretará, aunque fuera en el ámbito de quien la crea. ¿Qué ocurrirá entonces con el incrédulo, el que no se arriesga? "Si usted cree en lo que siempre ha creído, seguirá pensando como siempre ha pensado, y seguirá actuando como siempre lo ha hecho, y por lo mismo obtendrá los resultados que siempre ha obtenido" (Magomedova et al., 2020).

\section{Metodología}

Este artículo obedece a una investigación documental con un enfoque cualitativo, ya que se apoya en la revisión bibliográfica de documentación especializada sobre el tema estrategias de innovación y desarrollo económico. A nivel de conceptualización y contenidos alusivos a la temática planteada se hace una revisión exhaustiva sobre los términos: innovación, emprendimiento y sostenibilidad.

La técnica de recolección de información fue a través del análisis de documentos, mientras que el instrumento fue la matriz de inclusión y exclusión de las unidades de estudio. La fiabilidad se realizó a través de la triangulación y la interpretación mediante la categorización de datos.

A continuación, se presenta una tabla con la identificación de artículos incluidos y excluidos, por cada motor de búsqueda avanzada, según los criterios previamente establecidos.

\section{Tabla 1}

Artículos seleccionados, excluidos e incluidos para el análisis

\begin{tabular}{ccccc}
\hline \multirow{2}{*}{ Categoría } & \multicolumn{3}{c}{ Unidades de estudio encontradas por } & \multirow{2}{*}{ buscador } \\
\cline { 2 - 4 } & Researchgate & Dialnet & Scielo & \\
\hline $\begin{array}{c}\text { Artículos } \\
\text { seleccionados }\end{array}$ & 8 & 9 & 4 & 21 \\
\hline $\begin{array}{c}\text { Artículos } \\
\text { Excluidos }\end{array}$ & 6 & 2 & 3 & 11 \\
\hline $\begin{array}{c}\text { Artículos } \\
\text { Incluidos }\end{array}$ & 2 & 7 & 1 & 10 \\
\hline
\end{tabular}

Fuente: elaboración propia (2020).

De la búsqueda de información y revisión bibliográfica acerca de las variables innovación y desarrollo, así como desarrollo económico, se presenta a continuación el conjunto de derivaciones, contraposiciones, coincidencias y relaciones causales que dan lugar a la categorización de la investigación.

\section{Tabla 2}

Derivaciones, contraposiciones, coincidencias y relaciones causales que surgieron de las unidades de estudio

\begin{tabular}{|c|c|c|c|c|}
\hline Variables & Derivaciones & $\begin{array}{l}\text { Contrapo- } \\
\text { siciones }\end{array}$ & $\begin{array}{l}\text { Coinciden- } \\
\text { cias }\end{array}$ & $\begin{array}{c}\text { Rela- } \\
\text { ciones } \\
\text { Causales }\end{array}$ \\
\hline \multirow{3}{*}{$\begin{array}{c}\text { Innovación y } \\
\text { Desarrollo } \\
\text { Ycaza y Sánchez } \\
\text { (2016) } \\
\text { Camargo (2017) } \\
\text { Zurbriggen y } \\
\text { Lago (2020) }\end{array}$} & Globalización & \multirow{3}{*}{$\begin{array}{c}\text { Resistencia } \\
\text { al cambio }\end{array}$} & \multirow{3}{*}{$\begin{array}{l}\text { Resiliencia } \\
\text { Sociedad más } \\
\text { equilibrada y } \\
\text { equitativa }\end{array}$} & \multirow{2}{*}{$\begin{array}{l}\text { Obstácu- } \\
\text { los Cultu- } \\
\text { rales }\end{array}$} \\
\hline & $\begin{array}{l}\text { Regulación y } \\
\text { Desarrollo }\end{array}$ & & & \\
\hline & & & & $\begin{array}{c}\text { Nula } \\
\text { sustenta- } \\
\text { ción legal } \\
\text { para los } \\
\text { negocios }\end{array}$ \\
\hline
\end{tabular}




\begin{tabular}{ccccc}
\hline Variables & Derivaciones & $\begin{array}{c}\text { Contrapo- } \\
\text { siciones }\end{array}$ & $\begin{array}{c}\text { Coinciden- } \\
\text { cias }\end{array}$ & $\begin{array}{c}\text { Rela- } \\
\text { ciones } \\
\text { Causales }\end{array}$ \\
\hline $\begin{array}{c}\text { Desarrollo } \\
\text { Económico } \\
\text { Umaña et al } \\
(2018),\end{array}$ & $\begin{array}{c}\text { Emprendi- } \\
\text { miento }\end{array}$ & $\begin{array}{c}\text { Carencia } \\
\text { de prepara- } \\
\text { ción }\end{array}$ & $\begin{array}{c}\text { Búsqueda } \\
\text { de nuevas } \\
\text { estrategias }\end{array}$ & $\begin{array}{c}\text { Nuevos } \\
\text { Paradig- } \\
\text { mas }\end{array}$ \\
$\begin{array}{c}\text { González } \text { Ro- } \\
\text { mero (2018) } \\
\text { Eerina, (2020) }\end{array}$ & Económica & $\begin{array}{c}\text { Resistencia } \\
\text { al concepto } \\
\text { de Innova- } \\
\text { CEPAL (2015) }\end{array}$ & $\begin{array}{c}\text { Implemen- } \\
\text { tación de } \\
\text { nuevas ideas }\end{array}$ & $\begin{array}{c}\text { Recursos } \\
\text { y poten- } \\
\text { cialidad } \\
\text { de las } \\
\text { regiones }\end{array}$ \\
\hline
\end{tabular}

Fuente: elaboración propia (2020).

\section{Resultados}

Esta investigación de carácter documental, de una manera expedita aborda la innovación como una estrategia, un proceso, un cambio que actúa directamente ligado al desarrollo económico. No obstante, se puede observar en la revisión bibliográfica, la incidencia que posee para la innovación y desarrollo, el efecto de la globalización y la regulación, como tendencia económica mundial de anclaje y repercusión para una sociedad con mejores condiciones de vida, mediante la adaptación constante al cambio, venciendo los obstáculos culturales que determinan los procesos de innovación, integración y avance de la economía social.

Por su parte, la categoría desarrollo económico se concibe mediante el emprendimiento para lograr solidez económica, que requiere preparación y búsqueda de nuevas estrategias e ideas que permitan potenciar el recurso disponible en las regiones. Esta categoría está estrechamente ligada al emprendimiento con capacidad creativa y aglutina los diversos sectores de la sociedad para beneficiar al colectivo, para dar paso a una estrategia exitosa de desarrollo económico.

Se entiende la categoría emprendimiento, como la capacidad de asumir riesgos, de crear para crecer y renovar para transformar obstáculos y convertirlos en fortalezas. De igual forma, siendo la resiliencia una estrategia de adaptación a los cambios, es considerada como una de las posibles ventanas para abordar las dificultades y proponer soluciones. Quedan aprendizajes y nuevas propuestas de valor y una nueva manera de aproximarse a los consumidores en otra realidad social signada por la incertidumbre, el miedo, la angustia y el temor para enfrentar los cambios.

Estas afirmaciones se refuerzan, con lo señalado por García (2015), cuando dice que la innovación se manifiesta de diferentes formas para contribuir con el desarrollo económico, tal es el caso de la industria textil, basada en el proceso de transformación, desarrollo tecnológico y el cambio socio cultural luego de la adopción de nuevas tecnologías de información y comunicación, para la transformación de material reciclado, en insumo textil, contribuyendo de este modo, con el medio ambiente y el desarrollo económico del país.

Por su parte, opina Mendoza y García (2020), en cuanto a la innovación en el sector turismo, los prestadores de servicio y destinos turísticos deben ser objeto de diagnóstico, para lograr la toma de decisiones acertada como estrategia de reactivación económica, estabilidad laboral e incentivos, ante lo cual se hace necesario fomentar la promoción por redes sociales y la comercialización on line, como medio innovador de atracción masiva. Así mismo, Aguirre y Montalvo (2014), sostienen que las estrategias financieras utilizadas por las pymes en la crisis económica del 2000 al 2008, indican que el punto de partida para innovar, es un diagnóstico confiable, según las características de la empresa, para el logro de rentabilidad al enfrentar tiempo de crisis.

En cuanto a las estrategias de desarrollo económico, se plantean escenarios conducentes hacia el uso de tecnologías de información y comunicación, como medio de transporte de dinamismo e información que permite el flujo de patrones de conducta hacia la mejora de la calidad de vida, a través del componente de rentabilidad empresarial.

De este modo Montalbo-Coronel y Orozco-Santos (2020), resalta la importancia de la era digital y sus efectos en el mercado de equipos tecnológicos. Las medianas y pequeñas empresas deben innovar sus modelos económicos, para lograr un equilibrio en la cultura organizacional hacia lo digital, con habilidades tecnológicas, para lograr la competitividad, que requiere formación y capacitación del talento humano. Por su parte, Delgado y Álvarez (2020), y Magomedova et al. (2020), promueven la tecnología, dispositivo digital como herramienta de apoyo que debe ser utilizada como métodos y formas de aprender y formar al nuevo ciudadano. Un elemento aportado por Delgado y Álvarez (2020), es la importancia de las tecnologías como base sólida del sector bancario, basado en la incidencia de los canales electrónicos de gestión financiera.

\section{Conclusiones}

El presente estudio permitió estudiar estrategias innovadoras y desarrollo económico en tiempo de crisis, revelando la realidad actual en el ámbito del desarrollo económico y haciendo un análisis interpretativo de algunas consideraciones de revisión documental con elementos interpretativos y aportes sobre la temática tratada. En consecuencia, los conceptos de base resultan de una revisión bibliográfica consultada y a partir de fuentes fidedignas llegar a conclusiones coherentes sobre el hecho estudiado.

La innovación es un proceso complejo vinculado al emprendimiento, a la capacidad creativa y da paso a una estrategia exitosa de desarrollo económico. Por lo tanto, 
su radio de acción es el emprendimiento como estrategia exitosa de avance, que se complementa con la resiliencia como propuesta de valor. En cuanto al desarrollo económico, se considera la importancia del uso de tecnologías de información y comunicación, como tendencia gerencial de transmisión de conocimiento que promueve innovación y acelera cambios en el sector económico.

En este contexto, la investigación permitió concluir que las estrategias de innovadoras del mercado, tales como emprendimiento y creatividad, inciden positivamente en el desarrollo económico de los países, especialmente en tiempo de crisis, cuando es necesario reinventar las oportunidades y recursos disponibles para producir rentabilidad.

Por lo tanto, las estrategias de innovación para el desarrollo económico se deben establecer mediante un conjunto de actividades inherentes al emprendimiento y ser comunicadas por vía de las tecnologías de información y comunicación. 


\section{Referencias bibliográficas}

Aguirre, C. y Montalvo, B. (2014). Estudio de estrategias financieras para PYMES en tiempos de crisis en Pichincha. Editorial Quito: USFQ. http://repositorio.usfq.edu.ec/handle/23000/3367

Álvarez, C. (2013). Innovación, competitividad y nuevos modelos de negocio. Sinergia E InnovacióN, 1(08). http:// revistas.upc.edu.pe/index.php/sinergia/article/view/89

Blanco, I., Cruz Gallach, H., Martínez Moreno, R. \& Parés, M. (2016). El papel de la innovación social frente a la crisis. Ciudad Y Territorio Estudios Territoriales (CyTET), 48(188), 249-260. Recuperado a partir de https://recyt.fecyt.es/index. php/CyTET/article/view/76478

Camargo,J.,Contreras, F.y Jiménez, Y.(2017). Estado del arte dela innovación social: una miradaa laperspectivadeEuropa y Latinoamérica. Revista de Ciencias Humanas y Sociales, (82).https://dialnet.unirioja.es/servlet/articulo?codigo=6233641

Caridad, M., García, F., Martínez, S. y Morales, A. (2018). Bibliotecas y empoderamiento: servicios innovadores en un entorno de crisis. Revista Española De Documentación Científica, 41(2). https://doi.org/10.3989/redc.2018.2.1486

Delgado, J. y Álvarez, F. (2020). Análisis de la incidencia de los canales de los servicios financieros en el comportamiento de la bancarización en Ecuador. Periodo 2014 - 2018. Universidad Santiago de Guayaquil. http://repositorio.ucsg.edu.ec/ handle/3317/15830

Elisondo, R.y Donolo, D. (2013). Creatividad e Innovación. Cuadernos Artesanos de Educación. https://ri.conicet.gov.ar/ bitstream/handle/11336/123946/Libro-\%20Creatividad\%20e\%20Innovaci\%C3\%B3n.pdf?sequence=1

García, H. (2015), La innovación como estrategia de la industria textil. Transformando para subsistir: una alternativa para el Ecuador. Universidad de Guayaquil. Tiatemoani. Revista Académica de Investigación, (20). https://dialnet.unirioja. es/servlet/articulo?codigo $=7300876$

Goldenberg, M. (2018). Innovación en tiempos de crisis. Universidad de San Andrés. https://repositorio.udesa.edu.ar/ jspui/bitstream/10908/16710/1/\%5bP\%5d\%5bW\%5d\%20T.\%20G.\%20A.\%20y\%20C.\%20Goldenberg,\%20Micaela.pdf

González, J. y Romero, Y. (2018). La innovación tecnológica en las empresas y su impacto positivo dentro del Ecuador. Revista Observatorio de la Economía Latinoamericana. ht t p s://www.eumed.net/rev/oel/2018/03/innovacion-empresas-ecuador.html //hdl.handle.net/20.500.11763/oel1803innovacion-empresas-ecuador.

González, V. (2021). Creatividad y emprendimiento. Enfoque desde el talento humano. Repositorio Académico UPC. https://repositorioacademico.upc.edu.pe/handle/10757/655693

Guevara, J. (2020). Construyendo y adaptando modelos de negocio para la innovación en tiempos de crisis. Realidad Empresarial, (10), 2-6. https://doi.org/10.5377/reuca.v0i10.10568

Hernández, S. y Platero, M. (2012). La microempresa en tiempo de crisis. Análisis de la formación, la experiencia, la innovación. Revista de Estudios Cooperativos,(108). https://dialnet.unirioja.es/servlet/articulo?codigo=3982509

Magomedova, N., Roig, L. y Bastida, R. (2020). La innovación aplicada a la financiación de las empresas de la economía social. El caso de las inversiones de impacto. CIRIEC-Espana, (98). http://ciriec-revistaeconomia.es/wp-content/uploads/ CIRIEC_9805_Carreras_et_al.pdf

Martínez, G., Silva, F., Altamirano, M. y De la Torrea, F. (2021). Desarrollo tecnológico e innovación en México. Investigación y Pensamiento Crítico, 10. http://ojs.3ciencias.com/index.php/3c-empresa/article/view/1077

Mazin, A. (2021). Innovación en modelos de inversión y finanzas en tiempo de crisis. https://papers.ssrn.com/sol3/ papers.cfm?abstract_id=3840331 
Medina, L. (2020). Innovación Social: Conceptualización, desarrollo y situación actual en Europa y América Latina. http:// repositorio.ucsp.edu.pe/bitstream/20.500.12590/16377/1/MEDINA_CAPACOILA_LUC_INN.pdf

Méndez, R. (2016). Renovar economías urbanas en crisis: un debate actual sobre la innovación. Desenvolvimiento Regional en Debate, 6. (3). https://digital.csic.es/bitstream/10261/207005/1/renovar_economias.pdf

Mendoza, Á. y García, N. (2020). Estudio de pérdidas y estrategias de reactivación para el sector turístico por crisis sanitaria Covid en el destino del Manta. Ecuador. Revista internacional de Turismo. ISSN-e 2530-7134.

Montalbo-Coronel, L. y Orozco-Santos, C. (2020) Disrupción en tiempos de pandemia efectos en el mercado tecnológico en la provincia de Manaví-Ecuador. Revista científico - profesional, 5 (8), 353-375. https://dialnet.unirioja.es/servlet/ articulo?codigo $=7554379$

Munduate, M., Di Marco, D., Martínez, I. y Arenas, A. (2014). Reconstruyendo el diálogo social y promoviendo organizaciones inclusivas. Una herramienta para la innovación social en tiempos de crisis. Papeles del Psicólogo, 35 (2). https://idus.us.es/handle/11441/42420

Pimi, A. y Rafini, L. (2015). La empresa social en España y en Italia durante la crisis ¿un laboratorio de innovación económica y social? OBETS. Revista de Ciencias Sociales, 10(1), 127-158. doi:10.14198/OBETS2015.10.1.05

Pinedo, V y Pitre, R. (2017). Compilación de producción Primera edición - Primer Congreso Nacional de Ciencias Sociales y Humanas. La ciencia y la Educación Escenarios para la Construcción de Paz - Universidad de la Guajira (Compiladores Generales). https://www.researchgate.net/profile/Liliana-Contreras-Manrique/ publication/350021761_INFLUENCIA_DE_LA_INTELIGENCIA_EMOCIONAL_EN_EL_DESARROLLO_DEL_ PROYECTO_DE_VIDA_EN_JOVENES_VICTIMAS_DEL_CONFLICTO_ARMADO_INFLUENCIA_DE_LA_INTELIGENCIA_ EMOCIONAL_EN_EL_DESARROLLO_DEL_PROYECTO_DE_VIDA_EN_/links/604bd795a6fdcccfee 79c081/ INFLUENCIA-DE-LA-INTELIGENCIA-EMOCIONAL-EN-EL-DESARROLLO-DEL-PROYECTO-DE-VIDA-EN-JOVENESVICTIMAS-DEL-CONFLICTO-ARMADO-INFLUENCIA-DE-LA-INTELIGENCIA-EMOCIONAL-EN-EL-DESARROLLODEL-PROYECTO-DE-VIDA-EN.pdf\#page $=263$

Rodríguez, W. (2021). Sílabo de Innovación. Universidad Continental Repositorio Institucional Continental. https://repositorio.continental.edu.pe/handle/20.500.12394/8615

Sánchez, L. (2020). Innovación y creatividad en tiempos de crisis. Repositorio Instituciona RIñUPC. https://repositorio. unphu.edu.do/handle/123456789/3298

Subirats, J. y García, A. (2015). Innovación Social y políticas urbanas en España. Icaria Editorial. Primera Edición. https:// ddd.uab.cat/pub/llibres/2015/189796/innsocpol_a2015iSPA.pdf

Umaña, M., Villavicencio, J. y Gutiérrez, R. (2018). Factores de innovación que impactan en la competitividad global de la infraestructura en Latinoamérica. Comité Editorial, 154.https://www.researchgate.net/profile/Cesar_Sepulveda2/ publication/338540022_Analisis_sobre_las_causas_que_afectan_la_eficiencia_terminal_de_una_institucion_de educacion_superior_el_caso_de_la_carrera_de_Contador_Publico_del_IT_Mexicali/links/5e1a80a5a6fdcc28376ba61e/ Analisis-sobre-las-causas-que-afectan-la-eficiencia-terminal-de-una-institucion-de-educacion-superior-el-caso-de-lacarrera-de-Contador-Publico-del-IT-Mexicali.pdf\#page $=154$

Ycaza, D.y Sánchez,A. (2016). Factores de competitividad orientados a la pequeñay medianaempresa(PYME)en Latinoamérica. Revista San Gregorio, 2 (15). http://revista.sangregorio.edu.ec/index.php/REVISTASANGREGORIO/article/view/275

Villegas, R. (2021). Innovación, creatividad y desarrollo tecnológico de las Pymes. Revista Ciencias Empresariales y Sociales, 3 (1). https://publicacionescientificas.uces.edu.ar/index.php/empresarialesysociales/article/view/1043

Zurbriggen, C. y Lago, M. (2020). Innovación y co-creación: nuevos desafíos para las políticas públicas. Revista de gestión pública, 3(2). https://doi.org/10.22370/rgp.2014.3.2.2245 